OPEN ACCESS

Edited by:

Jens Staal,

Ghent University, Belgium

Reviewed by:

Volkan Cevik,

University of Bath, UK

Remco Stam,

Technische Universität München,

Germany

Xin $\mathrm{Li}$,

University of British Columbia,

Canada

*Correspondence:

Julio C. Vega-Arreguín

jvega@enes.unam.mx

Peter Moffett

peter.moffett@usherbrooke.ca

Specialty section:

This article was submitted to

Plant Microbe Interactions,

a section of the journal

Frontiers in Plant Science

Received: 25 October 2016 Accepted: 03 February 2017

Published: 15 February 2017

Citation:

Vega-Arreguín JC,

Shimada-Beltrán $H$,

Sevillano-Serrano $J$ and Moffett $P$

(2017) Non-host Plant Resistance

against Phytophthora capsici is

Mediated in Part by Members of the

$12 R$ Gene Family in Nicotiana spp.

Front. Plant Sci. 8:205

doi: 10.3389/fpls.2017.00205

\section{Non-host Plant Resistance against Phytophthora capsici Is Mediated in Part by Members of the I2 R Gene Family in Nicotiana spp.}

\author{
Julio C. Vega-Arreguín ${ }^{1,2 *}$, Harumi Shimada-Beltrán², Jacobo Sevillano-Serrano² and \\ Peter Moffett ${ }^{1,3 *}$ \\ ' Boyce Thompson Institute for Plant Research, Ithaca, NY, USA, ${ }^{2}$ Laboratorio de Ciencias Agrogenómicas, Escuela \\ Nacional de Estudios Superiores - León, Universidad Nacional Autónoma de Mexico, León, Mexico, ${ }^{3}$ Département de \\ Biologie, Faculté des Sciences, Université de Sherbrooke, Sherbrooke, QC, Canada
}

The identification of host genes associated with resistance to Phytophthora capsici is crucial to developing strategies of control against this oomycete pathogen. Since there are few sources of resistance to $P$. capsici in crop plants, non-host plants represent a promising source of resistance genes as well as excellent models to study P. capsici plant interactions. We have previously shown that non-host resistance to $P$. capsici in Nicotiana spp. is mediated by the recognition of a specific $P$. capsici effector protein, PcAvr3a1 in a manner that suggests the involvement of a cognate disease resistance $(R)$ genes. Here, we have used virus-induced gene silencing (VIGS) and transgenic tobacco plants expressing dsRNA in Nicotiana spp. to identify candidate $R$ genes that mediate non-host resistance to $P$. capsici. Silencing of members of the 12 multigene family in the partially resistant plant $N$. edwardsonii and in the resistant $N$. tabacum resulted in compromised resistance to $P$. capsici. VIGS of two other components required for $R$ gene-mediated resistance, EDS1 and SGT1, also enhanced susceptibility to $P$. capsici in N. edwardsonii, as well as in the susceptible plants $N$. benthamiana and $N$. clevelandii. The silencing of 12 family members in $N$. tabacum also compromised the recognition of PcAvr3a1. These results indicate that in this case, non-host resistance is mediated by the same components normally associated with race-specific resistance.

Keywords: Phytophthora capsici, Nicotiana, non-host resistance, $R$ gene, hypersensitive response

\section{INTRODUCTION}

Phytophthora capsici is a soil-borne oomycete that causes Phytophthora blight disease in many solanaceous and cucurbit plants worldwide (Hausbeck and Lamour, 2004; Tian and Babadoost, 2004; Lamour et al., 2012b). With more than 50 different plant species reported as hosts of $P$. capsici, and the increasing incidence of this disease, the development of resistance strategies is becoming an important challenge. In affected plants, the typical symptoms of the disease are wilting and a root and crown rot characterized by a dark brown stem lesion extending upward from the soil line. The infection can rapidly progress in appropriate conditions leading to the death of the plant. Despite increasing incidence of $P$. capsici, sources of genetic resistance in the most affected crop plants (pepper, eggplant, and cucurbits) are largely absent. Research on P. capsici has been enhanced by 
the sequencing of its genome (Joint Genome Institute ${ }^{1}$ ) and it is emerging as a model system for the study of host-oomycete interactions.

Plant disease resistance in which all accessions or cultivars of a plant species are resistant to all strains of a pathogen species is often referred to as non-host resistance. Non-host resistance allows most plants to resist most pathogens, although this broad classification is likely the manifestation of many different molecular mechanisms. This is in apparent contrast to racespecific, or gene-for-gene, disease resistance wherein dominant plant disease resistance $(R)$ genes confer resistance to specific pathogens encoding matching Avirulence (Avr) genes (Dangl and Jones, 2001). $R$ gene mediated resistance is an active process whereby recognition of pathogens results in the activation of multiple signaling pathways and often culminates a form of programmed cell death known as the hypersensitive response (HR) (Mur et al., 2008). Most Avr genes encode pathogen effector proteins and this phenomenon is now more commonly referred to as effector-triggered immunity (ETI) (Chisholm et al., 2006; Jones and Dangl, 2006). Plant $R$ genes are highly polymorphic both within and between populations (Clark et al., 2007; Ding et al., 2007; Yang et al., 2008), and effective $R$ genes are often introgressed into crop plants from wild relatives. Avr genes are also frequently polymorphic and $R$ genes may confer resistance to most pathogen or all pathogen races, isolates, or strains, or they may only be effective against a small subset. As such, some $R$ genes are durable over long periods of deployment, whereas others are quickly rendered ineffective due to newly emerged or introduced "resistance-breaking" strains (Harrison, 2002; McDonald and Linde, 2002; Parlevliet, 2002).

The majority of plant $R$ genes encode for NB-LRR (Nucleotide Binding Site-Leucine-Rich Repeat) proteins. There are three major classes of NB-LRR proteins, distinguished by the protein domain encoded at the $\mathrm{N}$ terminus; either a Toll and Interleukin1 Receptor homology domain (TIR; TIR-NB-LRRs), a loosely predicted coiled-coil domain (CC; CC-NB-LRRs) or a CC domain with homology to RPW8 (CC $C_{R}$ C $C_{R}-\mathrm{NB}-\mathrm{LRR}$ ) (Collier et al., 2011; Shao et al., 2016). NB-LRR proteins recognize effector/Avr proteins from all types of pathogens and several $R$ genes conferring resistance to members of the Phytophthora genus have been described including $R b / R p i B l b 1, R 1, R 3 b$, and $R 3 a$, which confer resistance to $P$. infestans (Ballvora et al., 2002; Song et al., 2003; van der Vossen et al., 2003; Armstrong et al., 2005; Li et al., 2011; Oh et al., 2014), and Rps1k, Rps4, and Rps6 which confer resistance to P. sojae (Sandhu et al., 2004; Gao et al., 2005). The potato resistance protein R3a was shown to recognize AVR3a from P. infestans (Armstrong et al., 2005; Bos et al., 2006) (Engelhardt et al., 2012). Also, silencing of Avr3a compromises $P$. infestans pathogenicity, indicating an important role of Avr3a in virulence (Bos et al., 2010).

The tomato 12 gene, a CC-NB-LRR R-gene that was shown to confer resistance to race 2 of Fusarium oxysporum $\mathrm{f}$ sp. lycopersici (Ori et al., 1997; Simons et al., 1998), is a homolog of the potato $R 3 a$ gene that confers resistance to $P$. infestans (Huang et al., 2004; Armstrong et al., 2005) as well as the $L$ genes

\footnotetext{
${ }^{1}$ http://genome.jgi.doe.gov/Phyca11/Phyca11.home.html
}

of pepper and the $N^{\prime}$ gene of tobacco, which confer resistance to tobamoviruses (Tomita et al., 2011; Sekine et al., 2012). Genes encoding I2-like proteins appear to be monophyletic clade specific to Solanaceous species, where they have expanded significantly in all the species investigated, including tobacco (Couch et al., 2006; Seo et al., 2016). Expression of the CC domain of I2-like proteins in Nicotiana benthamiana leaves is sufficient to induce an HR, whereas the LRR domain is thought to confer recognition specificity (Tomita et al., 2011; Sekine et al., 2012; Chapman et al., 2014; Hamel et al., 2016).

Plant signaling components important for defense against a variety of pathogens have also been identified. EDS1 (enhanced disease susceptibility 1) encodes a protein with homology to lipases that is an essential component of basal resistance to invasive biotrophs and certain hemibiotrophs (Parker et al., 1996; Wiermer et al., 2005). EDS1 is also required for the activity of the TIR-NBS-LRR class of $R$ genes (Aarts et al., 1998; Hu et al., 2005). The SGT1 (suppressor of the G2 allele of $s k p 1$ ) gene is essential for the function of most $R$ genes (Muskett and Parker, 2003; Azevedo et al., 2006). The SGT1 protein appears to play a role in NBLRR protein folding and accumulation (Holt et al., 2005; Azevedo et al., 2006) and elimination of SGT1 likely affects the activity of most or all NB-LRR proteins (Liu et al., 2002b; Peart et al., 2002b).

In this study, we used a candidate-gene approach to identify genes important for disease resistance to $P$. capsici. We have previously demonstrated that P. capsici Avr3al (PcAvr3a1) is recognized in several Nicotiana species and that such recognition is associated with resistance (Vega-Arreguin et al., 2014). Since PcAvr3a1 is a homolog of $P$. infestans Avr3a, which is recognized by the I2-like potato protein R3a gene, we hypothesized that a tobacco I2-like protein might confer resistance to $P$. capsici through the recognition of PcAvr3a1. Here, we have evaluated resistance to $P$. capsici in different Nicotiana species amenable to virus-induced gene silencing (VIGS) studies. Tobacco Rattle Virus (TRV) - based VIGS of I2-like, EDS1, and SGT1 was used to investigate their possible role in non-host resistance against $P$. capsici in several Nicotiana species. Transgenic N. tabacum expressing an $I 2$ hairpin construct for RNAi were also generated to evaluate the role of this gene in resistance. Differences in susceptibility to $P$. capsici were compared between silenced versus non-silenced plants upon inoculation with $P$. capsici zoospores. Our results indicate a role for 12 -like proteins in the recognition of PcAvr3a1 and resistance to P. capsici as well as a role for EDS1 and SGT1 in basal resistance in susceptible plants.

\section{RESULTS}

\section{Identification of a $P$. capsici Resistant Nicotiana Species Amenable to TRV-Based Virus-Induced Gene Silencing}

We have previously tested a number of Nicotiana species for their susceptibility or resistance to $P$. capsici based on application of zoospores to leaves (Vega-Arreguin et al., 2014). In order to use this information for reverse genetics, we tested a number of 
Nicotiana species for their amenability to TRV-based VIGS using the pTV00 vector (Ratcliff et al., 2001) carrying a fragment of the N. benthamiana Sulfur gene (encoding magnesium chelatase) (Kjemtrup et al., 1998; Lu et al., 2003). Supplementary Table S1 lists Nicotiana species amenable to TRV VIGS and Figure 1 shows a Sulfur-silenced N. edwardsonii plant 4 weeks post-infiltration.

To follow up these observations, we tested the response of a subset of VIGS-amenable Nicotiana species, N. benthamiana, $N$. clevelandii, and N. edwardsonii, to soil inoculation of zoospores. The New York isolate B1-3.1 of P. capsici was used for the infection assays. Four- to five-week-old plants were challenged with a $P$. capsici zoospore suspension and monitored for disease symptoms at 10 and 14 days post-inoculation (dpi). We found that $N$. benthamiana and $N$. clevelandii were highly susceptible to infection by $P$. capsici, similar to what was previously observed with detached leaves (Vega-Arreguin et al., 2014). At 10 and $14 \mathrm{dpi}$ all the N. benthamiana and N. clevelandii plants showed 80 to $100 \%$ of infection, whereas N. edwardsonii, a hybrid derived from a cross between $N$. glutinosa and N. clevelandii (Christie, 1969), showed only partial susceptibility with $14.7 \%$ of the plants showing $80-100 \%$ of infection at $14 \mathrm{dpi}$ (Table 1). Six other isolates of $P$. capsici from New York State: 06120-1, 0664-1, 0752-2, 0759-8, A3-3.1, and 8.2-1 were also tested on $N$. benthamiana with similar results (data not shown). Infection experiments with other Nicotiana species have shown that $N$. glutinosa, as well as N. tabacum, are resistant to P. capsici (Table 1 and Vega-Arreguin et al., 2014), but these species do not support TRV-based VIGS efficiently. Hence, the relatively weak susceptibility of $N$. edwardsonii to $P$. capsici makes it a
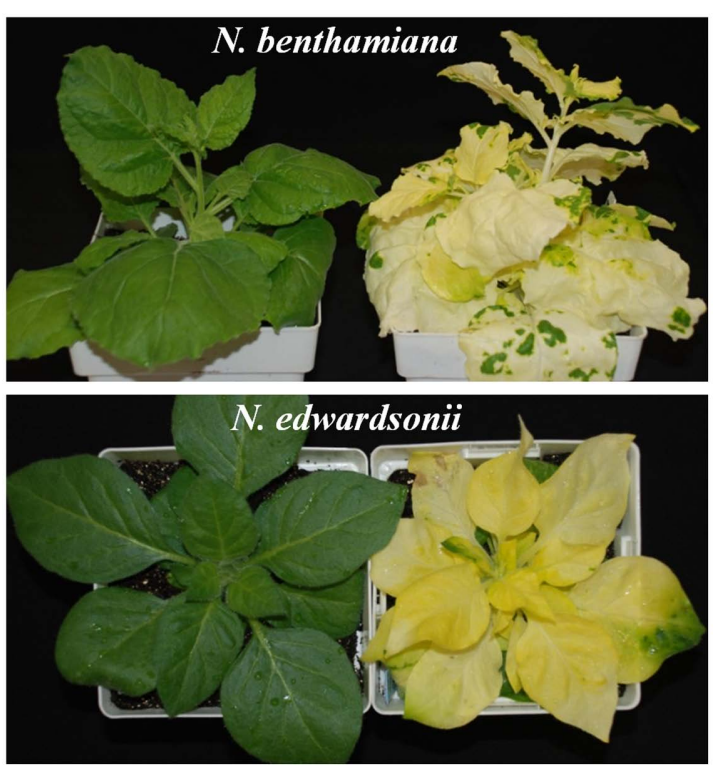

FIGURE 1 | Efficiency of TRV-based VIGS in $\mathbf{N}$. edwardsonii. Three-week-old N. edwardsonii and N. benthamiana plants were agro-infiltrated with Agrobacterium carrying a fragment of the $N$. benthamiana Sulfur (magnesium chelatase) gene cloned in TRV00 along with Agrobacterium carrying pBintra6 (RNA I) (right). Photographs were taken 4 weeks post-infiltration. good candidate for VIGS-based studies of resistance against P. capsici.

\section{Silencing of the Tobacco 12 Family Enhances Disease Susceptibility to $P$. capsici in N. edwardsonii and N. tabacum}

Since P. capsici Avr3al triggers an $\mathrm{HR}$ in tobacco and other Nicotiana species and such recognition correlates with resistance to $P$. capsici (Vega-Arreguin et al., 2014), and the tomato I2 protein can weakly recognize P. infestans Avr3a (Giannakopoulou et al., 2015), we hypothesized that a homolog of $I 2$ in tobacco could be mediating such resistance to $P$. capsici. To determine whether the tobacco $I 2$ gene family is involved in resistance to $P$. capsici, we used VIGS in $N$. edwardsonii and RNAi in stably transformed $N$. tabacum to silence most of the members of the family by targeting the highly conserved NBS region of I2. In a previous study, Couch et al. (2006) sampled 44 distinct I2-NBS sequences from tobacco. For VIGS of I2 we amplified and cloned in sense orientation into the pTRV2 vector (Liu et al., 2002a) a 840 bp sequence corresponding to the NBS region of $I 2$ from tobacco that shows high similarity with those reported previously (Couch et al., 2006) (Supplementary Figure S1). pTRV2-I2 was agroinfiltrated along with TRV1 in N. edwardsonii for VIGS of the I2 gene family. pTRV2Gus (Tameling and Baulcombe, 2007) and non-virus-infected plants served as controls. Figure $\mathbf{2 A}$ shows the I2-silenced $N$. edwardsonii plants before and after infection with $P$. capsici. The I2-silenced plants were more susceptible to $P$. capsici when compared with the control plants. At 10 dpi $30.8 \%$ of the plants did not present visible symptoms, compared to $61.5 \%$ in the control with pTRV2-Gus and $90 \%$ in the non-virus-infected plants (Figure 2B). At 14 dpi only $15.8 \%$ of the pTRV2-I2 infected plants were healthy and the controls with or without pTRV2-Gus presented 42.3 and $65 \%$ of healthy plants, respectively. Similar results were obtained when another sequence (also cloned in sense orientation into pTRV2) corresponding to the conserved NBS-I2 region chosen from the population of amplified PCR products was used for VIGS (data not shown), indicating that the silencing and phenotype derived thereof can occur with more than one NBS-I2 sequence.

It is noteworthy that TRV infection with pTRV2 carrying a fragment of Gus or RanGap2 (Tameling and Baulcombe, 2007) has some effect on $P$. capsici infection when compared with the "non-VIGS" (non-virus-infected) plants (Figures 2A,B) indicating that TRV infection itself appears to moderately enhance susceptibility to $P$. capsici. To test whether infection with a different virus has a similar response to the inoculation with $P$. capsici, plants were infected with Potato virus X (PVX) 3 weeks before inoculation with $P$. capsici. Contrary to that observed with TRV, plants infected with PVX were as resistant to P. capsici as the "non-VIGS" plants (Figure 2B). Thus, assuming that the silencing is specific, the enhanced susceptibility to $P$. capsici in $N$. edwardsonii is mainly due to the silencing of $I 2$.

To study the role of $\mathrm{I} 2$ against $P$. capsici in the resistant N. tabacum, we used the same NBS-I2 region used for VIGS 
TABLE 1 | P. capsici infection in $N$. benthamiana, $N$. clevelandii, $\boldsymbol{N}$. edwardsonii, and $\boldsymbol{N}$. tabacum.

\begin{tabular}{|c|c|c|c|c|c|c|c|c|}
\hline & \multicolumn{4}{|c|}{$10 \mathrm{dpi}$} & \multicolumn{4}{|c|}{$14 \mathrm{dpi}$} \\
\hline & $0^{*}$ & 1 & 2 & 3 & 0 & 1 & 2 & 3 \\
\hline N. benthamiana $(N=30)$ & $0^{a}$ & 0 & 0 & 100 & 0 & 0 & 0 & 100 \\
\hline N. clevelandii $(N=30)$ & 0 & 0 & 0 & 100 & 0 & 0 & 0 & 100 \\
\hline N. edwardsonii $(N=61)$ & 86.9 & 11.5 & 1.6 & 0 & 60.7 & 11.5 & 13.1 & 14.7 \\
\hline N. tabacum $(N=30)$ & 100 & 0 & 0 & 0 & 100 & 0 & 0 & 0 \\
\hline
\end{tabular}

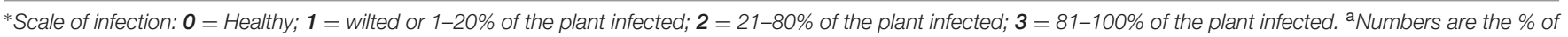
the total (N) plants tested.

to generate stable transformed plants with a hairpin construct to silence the $I 2$ gene family by RNAi. The NBS-I2 region was amplified and cloned into the pHellsgate vector (Wesley et al., 2001) in sense and antisense orientations. Transformation of $N$. tabacum plants with this construct generated 20 independent transformed lines. Transformed plants and detached leaves were inoculated either with a zoospore suspension and/or mycelium plugs of $P$. capsici, and infection development was monitored 2 to 6 days after inoculation. Figure 3 shows the detached leaves of wild-type (WT) and transformed plants inoculated with $P$. capsici. Detached leaves of $N$. tabacum transformed with an $I 2$ hairpin construct were more susceptible to $P$. capsici when compared to WT leaves (Figure 3). Similar results were obtained when detached leaves were inoculated with agar plugs containing mycelium of $P$. capsici (Supplementary Figure S3). Also, $I 2$ hairpin transgenic N. tabacum plants inoculated in the soil near the stem with $P$. capsici zoospores were more susceptible than the WT plants (Supplementary Figure S4). From these data, we conclude that a member of the multigene 12 family is important for resistance to P. capsici in N. edwardsonii and N. tabacum.

\section{Silencing of EDS1 Enhances Disease Susceptibility to $P$. capsici}

EDS1 has been shown to play a crucial role in basal defenses of plants and R-gene mediated resistance against different pathogens (Parker et al., 1996; Peart et al., 2002a; Bhattacharjee et al., 2011; Rietz et al., 2011; Moreau et al., 2012; Gao et al., 2014). To determine whether EDS1 is involved in resistance to $P$. capsici we silenced EDS1 in N. edwardsonii using VIGS. A pTRV2 vector containing a heterologous fragment of EDS1 gene from tobacco (Liu et al., 2002a) was inoculated by agroinfiltration along with TRV1 in 3-week-old N. edwardsonii plants. pTRV2-Gus was used as a control. Three to four weeks after infiltration with pTRV2EDS1 the plants were challenged with $P$. capsici zoospores and the infection was monitored at 10 and 14 dpi. Figures 2 A,B show the results of the EDS1 silencing experiment. At 10 and $14 \mathrm{dpi}$ EDS1-silenced plants showed enhanced disease susceptibility to $P$. capsici. The percentage of plants with no visible symptoms at 10 dpi decreased to $16.2 \%$ in pTRV2-EDS1 infiltrated plants, compared to 61.5 and $90 \%$ of healthy plants in the controls with or without pTRV2, respectively. At 14 dpi the healthy EDS1silenced plants represented only $2.7 \%$ of the total plants tested, compared to 42.3 and $65 \%$ in the controls. Thus, silencing of
EDS1 in N. edwardsonii clearly enhanced disease susceptibility to P. capsici.

\section{SILENCING OF SGT1 ENHANCES SUSCEPTIBILITY TO P. CAPSICI}

SGT1 (suppressor of the G2 allele of $s k p 1$ ) is required for resistance conferred by a number of R-genes (Peart et al., 2002b; Tor et al., 2002; Oh et al., 2014), and it was recently shown its role in pepper immunity against $P$. capsici upon interaction with SRC2-1 (Liu et al., 2016). We silenced this gene in $N$. benthamiana and $N$. clevelandii using VIGS. Silencing of SGT1 in N. edwardsonii, but not $N$. benthamiana and N. clevelandii, resulted in lethality (Supplementary Figure S2), indicating that SGT1 is not dispensable in this species, similar to VIGS results in tomato and double knock-out mutants of Arabidopsis SGT1a and SGT1b (Muskett and Parker, 2003; Bhattarai et al., 2007). Three weeks after the initiation of VIGS, plants were inoculated in the soil near the base of the plant with $2 \times 10^{5}$ zoospores of $P$. capsici B1-3.1. Silencing of SGT1 in N. benthamiana resulted in plants more susceptible to $P$. capsici than the vector-silenced plants as evidenced by a wilting phenotype caused by $P$. capsici observed at $2 \mathrm{dpi}$ in SGT1-silenced plants as opposed to $4 \mathrm{dpi}$ in control plants (Figures 4A,B,E). Similar results were obtained with $N$. clevelandii where infection progressed faster in SGT1silenced plants when compared to control plants (Figures 4C,D). These results indicate that SGT1 is important not only for $R$ gene mediated resistance but also for basal resistance responses to $P$. capsici.

\section{P. capsici Avr3a1-Triggered HR Is Compromised upon Silencing of $I 2$ in N. tabacum RNAi Transgenic Plants}

Since PcAvr3al triggers an HR in N. tabacum (Figure 5) and N. edwardsonii (Supplementary Figure S5) (Vega-Arreguin et al., 2014) we tested whether this PcAvr3a1-triggered HR is compromised in I2-silenced plants, either in N. tabacum stably transformed plants or in N. edwardsonii VIGS plants. Agrobacterium carrying PcAvr3al was infiltrated in leaves of I2silenced plants as well as in the control plants. HR was monitored at 5-8 dpi. Figure 5 shows the lack of PcAvr3al recognition in transgenic N. tabacum leaves agroinfiltrated with PcAvr3a1, whereas in N. tabacum WT leaves agroinfiltration of PcAvr3a1 
A
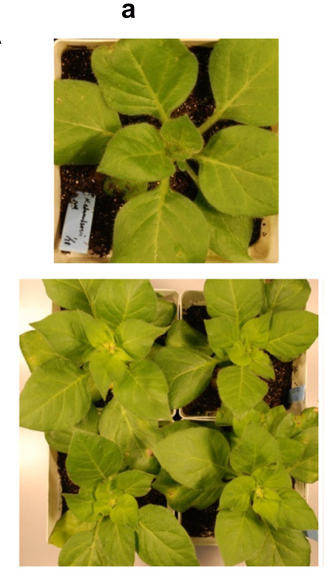

b

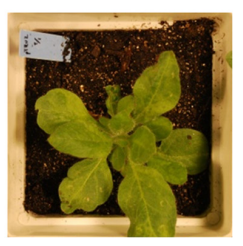

c

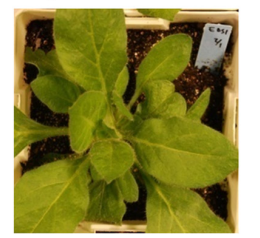

d

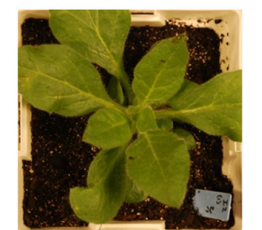

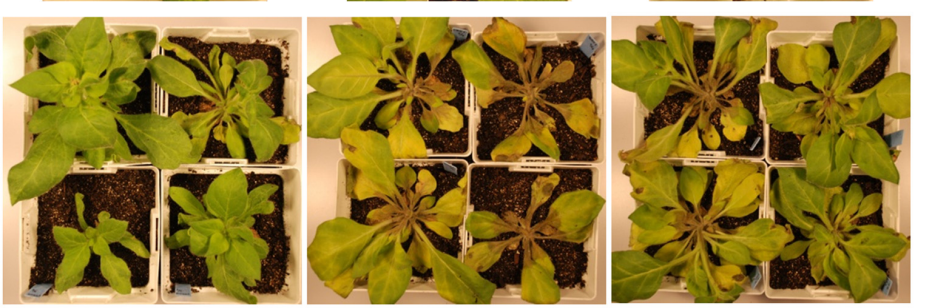

\section{政}

B

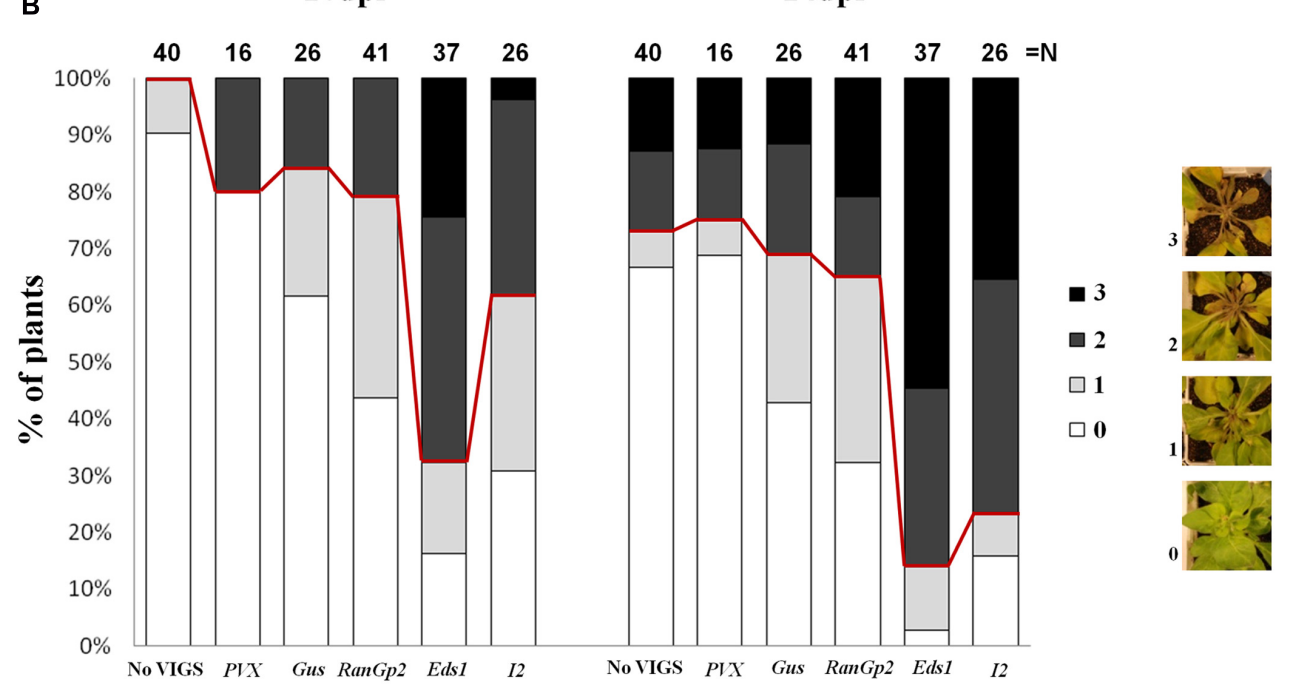

FIGURE 2 | Virus-induced gene silencing of EDS1 and $\mathbf{I}$ enhances susceptibility to $\boldsymbol{P}$. capsici in $\boldsymbol{N}$. edwardsonii. Three-week-old plants were infiltrated with Agrobacterium carrying PTRV2-EDS1 or PTRV2-12 along with Agrobacterium carrying TRV1. At 3 weeks post-infiltration plants were challenged with $5 \times 10^{5}$ zoospores of $P$. capsici inoculated in the soil near the base of the plant. (A) Top panel are agro-infiltrated plants right before inoculation with $P$. capsici. Bottom panel are the plants inoculated with P. capsici at 10 dpi. Control non-infiltrated plants (a), agro-infiltrated with pTRV2-Gus (b), pTRV2-EDS1 (c) or pTRV2-I2 (d). Experiments were repeated three times with similar results and representative pictures are presented. (B) Quantification of $P$. capsici infection in VIGSed N. edwardsonii plants. Controls were non-infiltrated plants or infiltrated with pTRV2-Gus, pTRV2-RanGp2, and PVX-based vector pGR107. Symptoms were monitored at 10 and 14 dpi according to the scale of infection as follows: $\mathbf{0}=$ Healthy; $\mathbf{1}=1-20 \%$ of the plant affected, Slightly Diseased; $\mathbf{2}=21-80 \%$ of the plant affected, Heavily Diseased; $\mathbf{3}=81-100 \%$ of the plant affected, Dead. N indicate the total number of plants tested from three independent experiments.

triggered a HR in the infiltration site. Agroinfiltration of Tobacco Mosaic Virus (TMV) p50 served as positive control since these tobacco plants contain the $\mathrm{N}$ resistant gene. In all cases, p50 triggered a $\mathrm{HR}$ in the infiltration site, indicating that the absence of HR upon agroinfiltration of PcAvr3al may be specifically a consequence of the silencing of the $I 2$ gene family and the lack of recognition of one of its members to PcAvr3al.

In N. edwardsonii VIGS plants, Agrobacterium carrying PcAvr3a1 was infiltrated in leaves of EDS1- and I2-silenced plants as well as in the VIGS control plants. HR was monitored at
5 dpi. Supplementary Figure S5 shows that the percentage of the infiltrated sites showing HR is not affected by the silencing of EDS1 and $I 2$ as compared with the controls, suggesting that PcAvr3al is being recognized by the remaining product of the silenced gene in $N$. edwardsonii, since VIGS usually reduces the level of the target transcript without completely knocking down the expression (Senthil-Kumar and Mysore, 2011). PcAvr3a does not trigger a visible HR upon agroinfiltration in N. benthamiana (Supplementary Figure S5), and silencing of SGT1 in N. edwardsonii resulted in lethality (Supplementary 


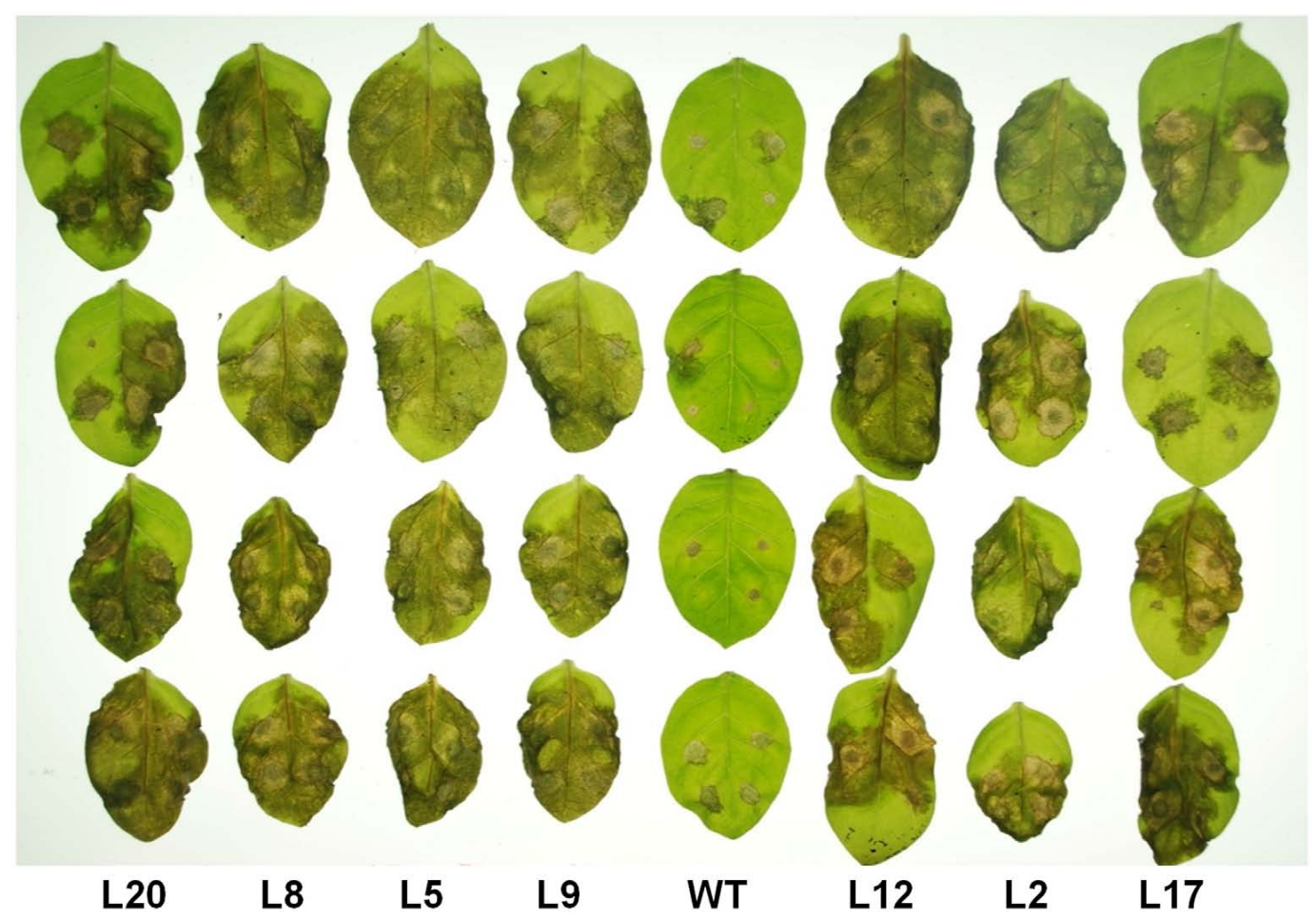

FIGURE 3 | Resistance to $\boldsymbol{P}$. capsici is compromised in transgenic tobacco leaves expressing a RNAi construct to silence $\mathbf{I} \mathbf{2}$ genes. Infection assay with P. capsici in transgenic tobacco lines expressing a hairpin of 12 was carried out in detached leaves of 4-week-old plants. Representative leaves from wild-type (WT) and different transgenic lines (L20, L8, L5, L9, L12, L2, and L17) inoculated with P. capsici zoospores are shown. Photographs were taken at 3 dpi.

Figure S2), thus the effect of silencing SGT1 in PcAvr3atriggered-HR was not assessed in these species. In N. clevelandii (which responds to PcAvr3al with a very weak necrosis, VegaArreguin et al., 2014), SGT1-silenced plants showed complete abolition of that PcAvr3a1-triggered necrosis (not shown), indicating that SGT1 is important for mediating recognition of PcAvr3a with its cognate $R$ gene.

\section{DISCUSSION}

Despite the economic importance of $P$. capsici, little is known about the genes involved in compatible or incompatible interactions with plants. Although some resistance genes have been described against $P$. infestans (for instance: $R 1, R B, R 3 a, R p i$ blb) (Ballvora et al., 2002; Song et al., 2003; van der Vossen et al., 2003; Armstrong et al., 2005; Oh et al., 2014) and P. sojae (Rps1k, Rp4, Rp6) (Sandhu et al., 2004; Gao et al., 2005), no cloned resistance genes against $P$. capsici have been reported. Non-host resistance to $P$. capsici in several Nicotiana species appears to be an active resistance mechanism as opposed to passive (true incompatibility) or PTI-mediated defense as supported by our previous findings on the correlation between resistance in several Nicotiana species with PcAvr3a1-triggered HR (Vega-Arreguin et al., 2014). As a first step toward understanding this resistance mechanism we have used VIGS and stable transformation with an RNAi construct in Nicotiana spp. to study the effect of silencing a multigene NB-LRR R family and two components (EDS1 and SGT1) required for disease resistance in plants. Our results demonstrate the viability of using silencing approaches to study the basis of non-host resistance to $P$. capsici in resistant plants. In addition, since silencing of EDS1 and SGT1 leads to even greater susceptibility, our approach can also be used to study the contribution of various components that participate in the basal level of resistance seen in susceptible plants. In principle then, this experimental approach should also be applicable to studying host factors involved in susceptibility and is complementary to the use of HIGS (Host-Induced Gene Silencing) in Nicotiana to study the role of $P$. capsici genes in infection (Vega-Arreguin et al., 2014).

The experimental system Nicotiana - P. capsici has become of particular interest as a model for $R$ gene and non-host resistance. Some advantages of this system are: (1) complete genome sequences are available for $N$. tabacum (Sierro et al., 2014), N. benthamiana (Bombarely et al., 2012) and P. capsici (Lamour et al., 2012a); (2) many Nicotiana species are particularly amenable to VIGS (Supplementary Table S1); (3) agro-infiltration of these plants is easy; and (4) most genes in Nicotiana are probably close enough in sequence that a gene sequence from one species can be used to silence in another without having to find the gene in the uncharacterized plants. In addition, VIGS can overcome gene function redundancy in multigene families by targeting a conserved region of the gene. Thus, most or all the members of the family will be silenced. Furthermore, TRV-based VIGS (Ratcliff et al., 2001; Liu et al., 2002a) has 

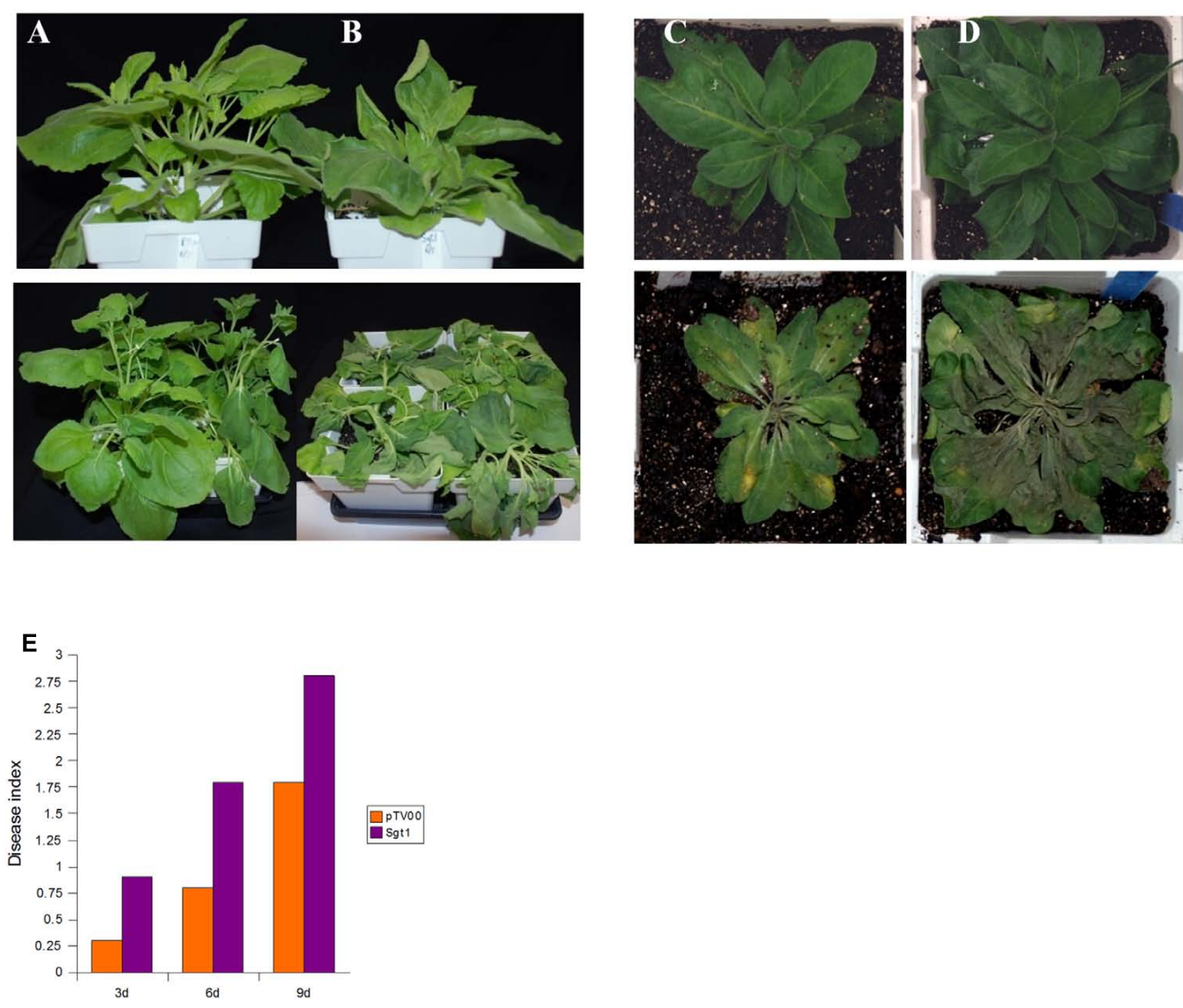

FIGURE 4 | Virus-induced gene silencing of SGT1 enhances susceptibility to $P$. capsici in $\boldsymbol{N}$. benthamiana and $\boldsymbol{N}$. clevelandii. Three-week-old N. benthamiana (A,B) and N. clevelandii (C,D) plants were subjected to TRV VIGS using TV-SGT1 (B,D) or pTV00 (A,C). At 3 weeks post-infiltration plants were challenged with $2 \times 10^{5}$ zoospores of $P$. capsici inoculated in the soil near the base of the plant. Symptoms were monitored at 3, 6, and 9 dpi. Top panels are VIGSed plants right before inoculation with P. capsici. Bottom panels are the plants inoculated with $P$. capsici at 3 dpi. (E) Disease index (DI $=[\Sigma$ (number of plants $\times$ category value)/total number of plants]) of the SGT1-silenced $P$. capsici-inoculated $N$. benthamiana plants at 3, 6, and 9 dpi. A representative experiment is presented from three independent replicates.

proven efficient in the identification of components involved in resistance signaling (Liu et al., 2002a, 2016; Peart et al., 2002a,b; Brigneti et al., 2004; Collier et al., 2011; Oh et al., 2014) and, as we have shown here, it can be an efficient tool to identify components of resistance signaling against soil-borne pathogens in Nicotiana species. Also, another relevant advantage of the Nicotiana - P. capsici system is the fact that different degrees of resistance to P. capsici can be observed in several Nicotiana species, for instance, $N$. benthamiana is susceptible, $N$. edwardsonii is partially susceptible and N. tabacum is resistant (Table 1). There are also cases of non-host resistance in Nicotiana species against $P$. capsici that might be different from what we have reported here, for example, $N$. plumbaginifolia is resistant to $P$. capsici but there is not a visible HR either with the pathogen or with PcAvr3a1 (Vega-Arreguin et al., 2014).

In this report, we have shown that silencing of the $I 2$ family resulted in enhanced susceptibility to $P$. capsici in N. edwardsonii and $N$. tabacum. Although further work will be required to identify the precise family member(s) that recognize PcAvr3a1, this represents the first NB-LRR $R$ gene family found to be involved in resistance to $P$. capsici. Silencing of $I 2$ compromised the PcAvr3a1-triggered HR in N. tabacum stably transformed with an RNAi construct (Figure 5). Although the HR was not compromised in $N$. edwardsonii VIGSed plants, VIGS of I2 nonetheless compromised resistance suggesting that the residual I2 product could be recognizing PcAvr3a1, which in this assay is likely much more abundant than when delivered from the pathogen. The compromise of resistance/Avr3a1 recognition by silencing the $I 2$ family in three Nicotiana species, as well as the common occurrence of the ability to recognize Avr3a1 (Vega-Arreguin et al., 2014) suggests that this recognition is mediated by a highly conserved I2 protein homolog that has been maintained in the Nicotiana genus. Cloning and characterization of these I2-like genes from resistant Nicotiana species will provide insights into non-host resistance to $P$. capsici, as well as opening up the possibility of transferring this "non-host" $R$ gene to susceptible Solanaceous crops.

EDS1 plays a role as a positive regulator of basal resistance but also in $R$ gene mediated resistance. EDS1 is required 

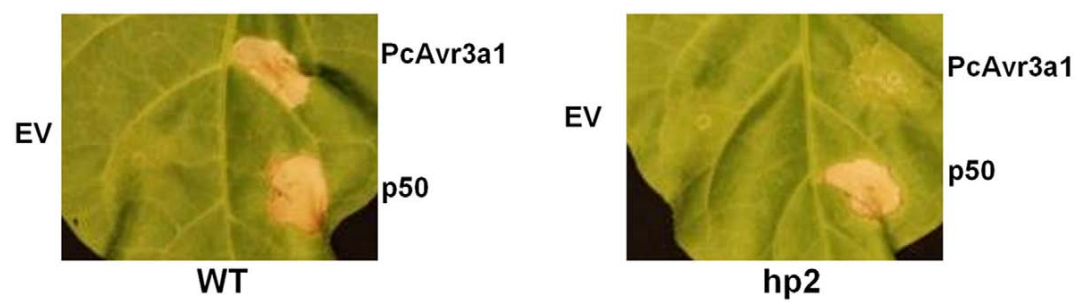

FIGURE 5 | PcAvr3a1-triggered HR in N. tabacum is compromised in transgenic I2-silenced plants. Leaves from 5- to 6-week-old N. tabacum (cv. Samsun NN) plants were infiltrated with Agrobacterium carrying either empty pBin61 (EV), or constructs expressing PcAvr3a1 or the TMV p50 protein, as indicated.

Photographs of representative leaves of WT (Left) and 12-hairpin transgenic (Right) plants were taken at 5 dpi.

for most cases of TIR-NB-LRR mediated resistance, although a few examples of EDS1 being required for CC-NB-LRR mediated resistance have been described. For instance, HRTmediated resistance against Turnip Crinkle Virus (ChandraShekara et al., 2004). EDS1 is also required for the function of RPW8, a non-NB-LRR protein, in resistance against powdery mildew (Xiao et al., 2005). Here, we noted that EDS1 silenced N. edwardsonii are more susceptible than the I2 silenced plants. At this point, we cannot unequivocally conclude that EDS1 is specifically for I2 mediated resistance against P. capsici, although this possibility also cannot be ruled out. Silencing of EDS1 may render the plant more susceptible overall to the oomycete, thus reducing the effectiveness of I2-like protein function. Further experiments would be required to test these possibilities.

A hemi-biotrophic lifestyle has been suggested to occur in several Phytophthora species and the transition from biotrophy to necrotrophy has been recently studied for P. capsici (Jupe et al., 2013) and P. infestans (Zuluaga et al., 2016) using transcriptome sequencing during the compatible interaction with tomato plants. In both cases, transcriptional changes associated with biotrophy and the subsequent switch to necrotrophy were described (Jupe et al., 2013; Zuluaga et al., 2016). Here, we found that EDS1 and SGT1, two important components of defense against biotrophs (Parker et al., 1996; Peart et al., 2002a,b; Collier et al., 2011; Liu et al., 2016) were also important for $R$ gene-mediated and basal resistance against $P$. capsici. We have shown in this study that silencing of heterologous EDS1 and SGT1 in diverse Nicotiana species enhances susceptibility to $P$. capsici. This is in contrast to what has been shown for the necrotroph Botrytis cinerea, where EDS1 and SGT1 are activated upon infection of $N$. benthamiana and the plants became resistant when these genes were silenced by VIGS (El Oirdi and Bouarab, 2007). These results suggest that the initial defenses against $P$. capsici function against the biotrophic phase of $P$. capsici. Indeed, we have previously reported that at very high inoculum, $P$. capsici can overcome the presumed $R$ gene-mediated, anti-biotrophic, resistance mechanisms of N. tabacum (Vega-Arreguin et al., 2014). These observations are pertinent to developing plants resistant to $P$. capsici as they suggest that once the pathogen switches to a necrotrophic phase, anti-biotrophic defense mechanisms will no longer be effective.

\section{MATERIALS AND METHODS}

\section{P. capsici Isolates and Zoospore Production}

P. capsici isolates B1-3.1, 06120-1, 0664-1, A3.1-1, 0752-2, 07581, 8.2-1 (kindly provided by Chris Smart, Geneva, NY, USA) were grown in $20 \%$ V8 vegetable juice agar (Campbells) complemented with 1 gr $\mathrm{CaCO} 3 / \mathrm{L}$ for 3-5 days in the dark at $26^{\circ} \mathrm{C}$. Sporulation was induced by growing three additional days under continuous fluorescent light. Release of zoospores was induced by flooding the cultures with sterile deionized water and incubated at $6^{\circ} \mathrm{C}$ for 30-60 min and then at room temperature for $1 \mathrm{~h}$. The amount of zoospores in the solution was determined by vortexing an aliquot of the zoospore solution for $30 \mathrm{~s}$ to induce encystment and counting the number of cysts with a hemocytometer.

\section{Plant Inoculation}

Plants were inoculated in the soil near the base of the plant with a zoospore suspension of $P$. capsici containing $5 \times 10^{5}$ zoospores. Plants were flooded immediately after inoculation for $24 \mathrm{~h}$ to allow free movement of zoospores and then maintained wet and incubated at $26^{\circ} \mathrm{C}$. P. capsici symptoms were recorded at 10 and 14 dpi for N. edwardsonii and at 3, 6, and 9 dpi for $N$. benthamiana and $N$. clevelandii. For recording symptoms, the following scale of infection ranging from 0 to 3 was used: $0=$ healthy, with no visible symptoms; $1=$ wilting or infection covering up to $20 \%$ of the plant; $2=$ infection covering up to $80 \%$ of the plant; $3=$ infection covering up to $100 \%$ of the plant. Since progression of the infection produced by $P$. capsici can differ depending on the plant species infected, we used this scale of infection because of its simplicity and reproducibility for recording infection in N. edwardsonii.

\section{Virus-Induced Gene Silencing}

Initial screening of VIGS-amenable species was performed using the pTV00 system as described (Ratcliff et al., 2001). Followup experiments using VIGS were performed as described using the pTRV2 vector (Liu et al., 2002a). Constructs pTRV2-EDS1, pTV00-SGT1, and pTRV2-Gus have been described before (Liu et al., 2002a; Peart et al., 2002b; Tameling and Baulcombe, 2007). To construct pTRV2-I2, a fragment of 840 nucleotides of the $I 2$ gene corresponding to the nucleotide binding site (NBS) region was amplified from $N$. tabacum DNA using primers $\mathrm{I} 2-2 \mathrm{~F}$ and 
I2-2R (Couch et al., 2006). A multiple alignment of 44 distinct NBS sequences derived from the tobacco $I 2$ multigene family (Couch et al., 2006) downloaded from the GenBank showed a high similarity (Supplementary Figure S1). Thus, for VIGS of I2 the PCR products were sub-cloned into pGEM-T easy vector (Promega) and a random clone was sequenced and subsequently cloned in sense orientation into the EcoRI site of the pTRV2 vector (Liu et al., 2002a).

Virus-induced gene silencing assays were carried out in N. edwardsonii, N. benthamiana, and N. clevelandii. Three-weekold plants were infiltrated with the Agrobacterium strain GV3101 and C58C1 harboring TRV1 along with Agrobacterium harboring pTRV2-EDS1, pTRV2-I2, or pTV00-SGT1. Agroinfiltration of VIGS constructs was carried out as previously described (Collier et al., 2011). Plants were kept in the greenhouse for 3 weeks after infiltration and then moved to a restricted-access confinement facility for inoculation with $P$. capsici.

\section{Transgenic Plants}

Transformation of N. tabacum with the RNAi construct NtI2hp was performed at the Plant Transformation Core Laboratory of the Boyce Thompson Institute for Plant Research, Ithaca, NY, USA. Twenty independent lines were obtained and tested for resistance to $P$. capsici. For plant transformation, the binary RNAi construct NtI2hp was generated using the RNAi gateway vector pHellsgate (Wesley et al., 2001) and introduced into Agrobacterium strain EHA105 by electroporation.

\section{AUTHOR CONTRIBUTIONS}

Conceived and designed the experiments: JV-A and PM. Performed the experiments: JV-A and JS-S. Analyzed the data: JV-A, HS-B, JS-S, and PM. Contributed reagents/materials/analysis tools: JV-A, HS-B, and PM. Wrote the paper: JV-A and PM.

\section{FUNDING}

Funding for this project was provided by the New York State Department of Agriculture and Markets. We also thank UNAMDGAPA-PAPIIT (IA203313 - IN214917) and CONCYTEG for partial funding of this project.

\section{REFERENCES}

Aarts, N., Metz, M., Holub, E., Staskawicz, B. J., Daniels, M. J., and Parker, J. E. (1998). Different requirements for EDS1 and NDR1 by disease resistance genes define at least two $\mathrm{R}$ gene-mediated signaling pathways in Arabidopsis. Proc. Natl. Acad. Sci. U.S.A. 95, 10306-10311. doi: 10.1073/pnas.95.17.10306

Armstrong, M. R., Whisson, S. C., Pritchard, L., Bos, J. I., Venter, E., Avrova, A. O., et al. (2005). An ancestral oomycete locus contains late blight avirulence gene Avr3a, encoding a protein that is recognized in the host cytoplasm. Proc. Natl. Acad. Sci. U.S.A. 102, 7766-7771. doi: 10.1073/pnas.0500113102

\section{ACKNOWLEDGMENTS}

We are grateful to Chris Smart and Bill Fry for P. capsici isolates and helpful discussions. We thank Abubakar Jalloh for technical assistance.

\section{SUPPLEMENTARY MATERIAL}

The Supplementary Material for this article can be found online at: http://journal.frontiersin.org/article/10.3389/fpls.2017.00205/ full\#supplementary-material

FIGURE S1 | Alignment of the tobacco I2-NBS sequence used for VIGS in $N$. edwardsonii and for transformation of $N$. tabacum plants with the 44 sequences of the NBS region of $\mathbf{2}$ homologs from tobacco reported previously (Couch et al., 2006). The alignment showed an overall similarity of $78.5 \%$. One of the more conserved blocks of the alignment (containing at least 25 identical nucleotides in all sequences) is presented to show the potential of VIGS and RNAi to silence most of the members of the 12 family.

FIGURE S2 | VIGS of SGT1 in N. edwardsonii results in lethality. Three-week-old plants were infiltrated with Agrobacterium carrying TV-SGT1 or pTV00 along with Agrobacterium carrying pBintra6 (RNA I), as indicated. Non-TRV-infected plants are shown for comparison. Photographs were taken 3 weeks after initiation of VIGS.

FIGURE S3 | Resistance to $P$. capsici is compromised in transgenic tobacco leaves expressing a RNAi construct to silence $\mathbf{1 2}$ genes. Infection assay with $P$. capsici in transgenic tobacco lines expressing a hairpin of 12 was carried out in detached leaves of 4-week-old plants. The graph shows the infection size (diameter) in leaves from wild-type (WT) and the different transgenic lines ( L1-L20) inoculated with P. capsici mycelium. Data was collected at $2 \mathrm{dpi}$ and the average of measurements from three to six independent experiments is shown. Error bars represent standard errors.

FIGURE S4 | Resistance to $P$. capsici is compromised in transgenic tobacco plants expressing a RNAi construct to silence $\mathbf{1 2}$ genes. Infection assay with $P$. capsici in transgenic tobacco lines expressing a hairpin of 12 was carried out in 2-week-old plants. The graph shows the ratio of infected plants/total inoculated plants. This is a representative experiment in which 18 to 24 plants were used of each of the WT and the different transgenic lines ( L1- L20) and the data represents the absolute number of plants tested. Plants were inoculated in the soil near the stem with a suspension of $18 \times 10^{4}$ zoospores of $P$. capsici. Data was collected at 6 dpi.

FIGURE S5 | $P$. capsici Avr3a-triggered HR in $N$. edwardsonii is not compromised in EDS1- and I2-silenced plants. (A) Leaves of $N$. edwardsonii and $N$. benthamiana VIGSed for 12 were agro-infiltrated with Avr3a1 or empty vector (pBin61). Photographs were taken at 5 dpi. (B) Percentages of infiltrated sites showing HR in non-VIGSed $N$. edwardsonii plants and plants VIGSed with TRV carrying inserts of GUS, EDS1- and 12. HRs were scored at 5-8 dpi and error bars indicate standard deviation from three independent experiments.

Azevedo, C., Betsuyaku, S., Peart, J., Takahashi, A., Noel, L., Sadanandom, A., et al. (2006). Role of SGT1 in resistance protein accumulation in plant immunity. EMBO J. 25, 2007-2016. doi: 10.1038/sj.emboj.7601084

Ballvora, A., Ercolano, M. R., Weiss, J., Meksem, K., Bormann, C. A., Oberhagemann, P., et al. (2002). The R1 gene for potato resistance to late blight (Phytophthora infestans) belongs to the leucine zipper/NBS/LRR class of plant resistance genes. Plant J. 30, 361-371. doi: 10.1046/j.1365-313X.2001.01292.x

Bhattacharjee, S., Halane, M. K., Kim, S. H., and Gassmann, W. (2011). Pathogen effectors target Arabidopsis EDS1 and alter its interactions with immune regulators. Science 334, 1405-1408. doi: 10.1126/science.1211592 
Bhattarai, K. K., Li, Q., Liu, Y., Dinesh-Kumar, S. P., and Kaloshian, I. (2007). The MI-1-mediated pest resistance requires Hsp90 and Sgt1. Plant Physiol. 144, 312-323. doi: 10.1104/pp.107.097246

Bombarely, A., Rosli, H. G., Vrebalov, J., Moffett, P., Mueller, L. A., and Martin, G. B. (2012). A draft genome sequence of Nicotiana benthamiana to enhance molecular plant-microbe biology research. Mol. Plant Microbe Interact. 25, 1523-1530. doi: 10.1094/MPMI-06-12-0148-TA

Bos, J. I., Armstrong, M. R., Gilroy, E. M., Boevink, P. C., Hein, I., Taylor, R. M., et al. (2010). Phytophthora infestans effector AVR3a is essential for virulence and manipulates plant immunity by stabilizing host E3 ligase CMPG1. Proc. Natl. Acad. Sci. U.S.A. 107, 9909-9914. doi: 10.1073/pnas.0914408107

Bos, J. I., Kanneganti, T. D., Young, C., Cakir, C., Huitema, E., Win, J., et al. (2006). The C-terminal half of Phytophthora infestans RXLR effector AVR3a is sufficient to trigger R3a-mediated hypersensitivity and suppress INF1-induced cell death in Nicotiana benthamiana. Plant J. 48, 165-176. doi: 10.1111/j.1365-313X.2006. 02866.x

Brigneti, G., Martin-Hernandez, A. M., Jin, H., Chen, J., Baulcombe, D. C., Baker, B., et al. (2004). Virus-induced gene silencing in Solanum species. Plant J. 39, 264-272. doi: 10.1111/j.1365-313X.2004.02122.x

Chandra-Shekara, A. C., Navarre, D., Kachroo, A., Kang, H. G., Klessig, D., and Kachroo, P. (2004). Signaling requirements and role of salicylic acid in HRTand rrt-mediated resistance to turnip crinkle virus in Arabidopsis. Plant J. 40, 647-659. doi: 10.1111/j.1365-313X.2004.02241.x

Chapman, S., Stevens, L. J., Boevink, P. C., Engelhardt, S., Alexander, C. J., Harrower, B., et al. (2014). Detection of the virulent form of AVR3a from Phytophthora infestans following artificial evolution of potato resistance gene R3a. PLoS ONE 9:e110158. doi: 10.1371/journal.pone.0110158

Chisholm, S. T., Coaker, G., Day, B., and Staskawicz, B. J. (2006). Host-microbe interactions: shaping the evolution of the plant immune response. Cell 124, 803-814. doi: 10.1016/j.cell.2006.02.008

Christie, S. R. (1969). Nicotiana hybrid developed as a host for plant viruses. Plant Dis. Rep. 53, 939-941.

Clark, R. M., Schweikert, G., Toomajian, C., Ossowski, S., Zeller, G., Shinn, P., et al. (2007). Common sequence polymorphisms shaping genetic diversity in Arabidopsis thaliana. Science 317, 338-342. doi: 10.1126/science.1138632

Collier, S. M., Hamel, L. P., and Moffett, P. (2011). Cell death mediated by the N-terminal domains of a unique and highly conserved class of NB-LRR protein. Mol. Plant Microbe Interact. 24, 918-931. doi: 10.1094/MPMI-03-11-0050

Couch, B. C., Spangler, R., Ramos, C., and May, G. (2006). Pervasive purifying selection characterizes the evolution of I2 homologs. Mol. Plant Microbe Interact. 19, 288-303. doi: 10.1094/MPMI-19-0288

Dangl, J. L., and Jones, J. D. (2001). Plant pathogens and integrated defence responses to infection. Nature 411, 826-833. doi: 10.1038/35081161

Ding, J., Zhang, W., Jing, Z., Chen, J. Q., and Tian, D. (2007). Unique pattern of R-gene variation within populations in Arabidopsis. Mol. Genet. Genomics 277, 619-629. doi: 10.1007/s00438-007-0213-5

El Oirdi, M., and Bouarab, K. (2007). Plant signalling components EDS1 and SGT1 enhance disease caused by the necrotrophic pathogen Botrytis cinerea. New Phytol. 175, 131-139. doi: 10.1111/j.1469-8137.2007.02086.x

Engelhardt, S., Boevink, P. C., Armstrong, M. R., Ramos, M. B., Hein, I., and Birch, P. R. J. (2012). Relocalization of late blight resistance protein R3a to endosomal compartments is associated with effector recognition and required for the immune response. Plant Cell 24, 5142-5158. doi: 10.1105/tpc.112. 104992

Gao, F., Dai, R., Pike, S. M., Qiu, W., and Gassmann, W. (2014). Functions of EDS1like and PAD4 genes in grapevine defenses against powdery mildew. Plant Mol. Biol. 86, 381-393. doi: 10.1007/s11103-014-0235-4

Gao, H., Narayanan, N. N., Ellison, L., and Bhattacharyya, M. K. (2005). Two classes of highly similar coiled coil-nucleotide binding-leucine rich repeat genes isolated from the Rps1-k locus encode Phytophthora resistance in soybean. Mol. Plant Microbe Interact. 18, 1035-1045. doi: 10.1094/MPMI-18-1035

Giannakopoulou, A., Steele, J. F. C., Segretin, M. E., Bozkurt, T., Zhou, J., Robatzek, S., et al. (2015). Tomato I2 immune receptor can be engineered to confer partial resistance to the oomycete Phytophthora infestans in addition to the fungus Fusarium oxysporum. Mol. Plant-Microbe Interact. 28, 1316-1329. doi: 10.1094/MPMI-07-15-0147-R

Hamel, L. P., Sekine, K. T., Wallon, T., Sugiwaka, Y., Kobayashi, K., and Moffett, P. (2016). The chloroplastic protein THF1 interacts with the coiled-coil domain of the disease resistance protein N' and regulates light-dependent cell death. Plant Physiol. 171, 658-674. doi: 10.1104/pp.16.00234

Harrison, B. D. (2002). Virus variation in relation to resistance-breaking in plants. Euphytica 124, 181-192. doi: 10.1007/s00705-014-2097-0

Hausbeck, M. K., and Lamour, K. H. (2004). Phytophtora capsici vegetable crops: research progress and management challenges. Plant Dis. 88, 1292-1303. doi: 10.1094/PDIS.2004.88.12.1292

Holt, B. F. III, Belkhadir, Y., and Dangl, J. L. (2005). Antagonistic control of disease resistance protein stability in the plant immune system. Science 309, 929-932. doi: 10.1126/science.1109977

Hu, G., deHart, A. K., Li, Y., Ustach, C., Handley, V., Navarre, R., et al. (2005). EDS1 in tomato is required for resistance mediated by TIR-class R genes and the receptor-like R gene Ve. Plant J. 42, 376-391. doi: 10.1111/j.1365-313X.2005. 02380.x

Huang, S., Vleeshouwers, V. G., Werij, J. S., Hutten, R. C., van Eck, H. J., Visser, R. G., et al. (2004). The R3 resistance to Phytophthora infestans in potato is conferred by two closely linked R genes with distinct specificities. Mol. Plant Microbe Interact. 17, 428-435. doi: 10.1094/MPMI.2004.17.4.428

Jones, J. D., and Dangl, J. L. (2006). The plant immune system. Nature 444, 323-329. doi: 10.1038/nature05286

Jupe, J., Stam, R., Howden, A. J., Morris, J. A., Zhang, R., Hedley, P. E., et al. (2013). Phytophthora capsici-tomato interaction features dramatic shifts in gene expression associated with a hemi-biotrophic lifestyle. Genome Biol. 14:R63. doi: 10.1186/gb-2013-14-6-r63

Kjemtrup, S., Sampson, K. S., Peele, C. G., Nguyen, L. V., Conkling, M. A., Thompson, W. F., et al. (1998). Gene silencing from plant DNA carried by a Geminivirus. Plant J. 14, 91-100. doi: 10.1046/j.1365-313X.1998.00101.x

Lamour, K. H., Mudge, J., Gobena, D., Hurtado-Gonzales, O. P., Schmutz, J., Kuo, A., et al. (2012a). Genome sequencing and mapping reveal loss of heterozygosity as a mechanism for rapid adaptation in the vegetable pathogen Phytophthora capsici. Mol. Plant Microbe Interact. 25, 1350-1360. doi: 10.1094/ MPMI-02-12-0028-R

Lamour, K. H., Stam, R., Jupe, J., and Huitema, E. (2012b). The oomycete broadhost-range pathogen Phytophthora capsici. Mol. Plant Pathol. 13, 329-337. doi: 10.1111/j.1364-3703.2011.00754.x

Li, G., Huang, S., Guo, X., Li, Y., Yang, Y., Guo, Z., et al. (2011). Cloning and characterization of $\mathrm{r} 3 \mathrm{~b}$; members of the $\mathrm{r} 3$ superfamily of late blight resistance genes show sequence and functional divergence. Mol. Plant Microbe Interact. 24, 1132-1142. doi: 10.1094/MPMI-11-10-0276

Liu, Y., Schiff, M., Marathe, R., and Dinesh-Kumar, S. P. (2002a). Tobacco Rar1, EDS1 and NPR1/NIM1 like genes are required for N-mediated resistance to tobacco mosaic virus. Plant J. 30, 415-429.

Liu, Y., Schiff, M., Serino, G., Deng, X- W., and Dinesh-Kumar, S. P. (2002b). Role of SCF ubiquitin-ligase and the COP9 signalosome in the N gene-mediated resistance response to tobacco mosaic virus. Plant Cell 14, 1483-1496. doi: $10.1105 /$ tpc. 002493

Liu Z-q, Liu Y-y, Shi, L-p, Yang, S., Shen, L., Yu H-x, et al. (2016). SGT1 is required in PcINF1/SRC2-1 induced pepper defense response by interacting with SRC2-1. Sci. Rep. 6:21651. doi: 10.1038/srep21651

Lu, R., Martin-Hernandez, A. M., Peart, J. R., Malcuit, I., and Baulcombe, D. C. (2003). Virus-induced gene silencing in plants. Methods 30, 296-303. doi: 10. 1016/S1046-2023(03)00037-9

McDonald, B. A., and Linde, C. (2002). The population genetics of plant pathogens and breeding strategies for durable resistance. Euphytica 124, 163-180. doi: 10.1023/A:1015678432355

Moreau, M., Degrave, A., Vedel, R., Bitton, F., Patrit, O., Renou, J. P., et al. (2012). EDS1 contributes to nonhost resistance of Arabidopsis thaliana against Erwinia amylovora. Mol. Plant Microbe Interact. 25, 421-430. doi: 10.1094/MPMI-0511-0111

Mur, L. A., Kenton, P., Lloyd, A. J., Ougham, H., and Prats, E. (2008). The hypersensitive response; the centenary is upon us but how much do we know? J. Exp. Bot. 59, 501-520. doi: 10.1093/jxb/erm239

Muskett, P., and Parker, J. (2003). Role of SGT1 in the regulation of plant R gene signalling. Microbes Infect. 5, 969-976. doi: 10.1016/S1286-4579(03)00183-7

Oh, S. K., Kwon, S. Y., and Choi, D. (2014). Rpi-blb2-mediated hypersensitive cell death caused by Phytophthora infestans AVRblb2 requires SGT1, but not EDS1, NDR1, salicylic acid-, jasmonic acid-, or ethylene-mediated signaling. Plant Pathol. J. 30, 254-260. doi: 10.5423/PPJ.OA.03.2014.0027 
Ori, N., Eshed, Y., Paran, I., Presting, G., Aviv, D., Tanksley, S., et al. (1997). The I2C family from the wilt disease resistance locus $\mathrm{I} 2$ belongs to the nucleotide binding, leucine-rich repeat superfamily of plant resistance genes. Plant Cell 9, 521-532. doi: 10.1105/tpc.9.4.521

Parker, J. E., Holub, E. B., Frost, L. N., Falk, A., Gunn, N. D., and Daniels, M. J. (1996). Characterization of eds1, a mutation in Arabidopsis suppressing resistance to Peronospora parasitica specified by several different RPP genes. Plant Cell 8, 2033-2046. doi: 10.1105/tpc.8.11.2033

Parlevliet, J. E. (2002). Durability of resistance against fungal, bacterial and viral pathogens; present situation. Euphytica 124, 147-156. doi: 10.1023/A: 1015601731446

Peart, J. R., Cook, G., Feys, B. J., Parker, J. E., and Baulcombe, D. C. (2002a). An EDS1 orthologue is required for $\mathrm{N}$-mediated resistance against tobacco mosaic virus. Plant J. 29, 569-579.

Peart, J. R., Lu, R., Sadanandom, A., Malcuit, I., Moffett, P., Brice, D. C., et al. (2002b). Ubiquitin ligase-associated protein SGT1 is required for host and nonhost disease resistance in plants. Proc. Natl. Acad. Sci. U.S.A. 99, 10865-10869. doi: 10.1073/pnas.152330599

Ratcliff, F., Martin-Hernandez, A. M., and Baulcombe, D. C. (2001). Technical advance. Tobacco rattle virus as a vector for analysis of gene function by silencing. Plant J. 25, 237-245. doi: 10.1046/j.0960-7412.2000. 00942.x

Rietz, S., Stamm, A., Malonek, S., Wagner, S., Becker, D., Medina-Escobar, N., et al. (2011). Different roles of enhanced disease susceptibility1 (EDS1) bound to and dissociated from phytoalexin deficient4 (PAD4) in Arabidopsis immunity. New Phytol. 191, 107-119. doi: 10.1111/j.1469-8137.2011.03675.x

Sandhu, D., Gao, H., Cianzio, S., and Bhattacharyya, M. K. (2004). Deletion of a disease resistance nucleotide-binding-site leucine-rich-repeat-like sequence is associated with the loss of the Phytophthora resistance gene Rps4 in soybean. Genetics 168, 2157-2167. doi: 10.1534/genetics.104.032037

Sekine, K. T., Tomita, R., Takeuchi, S., Atsumi, G., Saitoh, H., Mizumoto, H., et al. (2012). Functional differentiation in the leucine-rich repeat domains of closely related plant virus-resistance proteins that recognize common avr proteins. Mol. Plant Microbe Interact. 25, 1219-1229. doi: 10.1094/MPMI-1111-0289

Senthil-Kumar, M., and Mysore, K. S. (2011). New dimensions for VIGS in plant functional genomics. Trends Plant Sci. 16, 656-665. doi: 10.1016/j.tplants.2011. 08.006

Seo, E., Kim, S., Yeom, S. I., and Choi, D. (2016). Genome-wide comparative analyses reveal the dynamic evolution of nucleotide-binding leucine-rich repeat gene family among Solanaceae plants. Front. Plant Sci. 7:1205. doi: 10.3389/fpls. 2016.01205

Shao, Z. Q., Xue, J. Y., Wu, P., Zhang, Y. M., Wu, Y., Hang, Y. Y., et al. (2016). Large-scale analyses of angiosperm nucleotide-binding site-leucine-rich repeat genes reveal three anciently diverged classes with distinct evolutionary patterns. Plant Physiol. 170, 2095-2109. doi: 10.1104/pp.15.01487

Sierro, N., Battey, J. N. D., Ouadi, S., Bakaher, N., Bovet, L., Willig, A., et al. (2014). The tobacco genome sequence and its comparison with those of tomato and potato. Nat. Commun. 5:3833. doi: 10.1038/ncomms4833

Simons, G., Groenendijk, J., Wijbrandi, J., Reijans, M., Groenen, J., Diergaarde, P., et al. (1998). Dissection of the fusarium I2 gene cluster in tomato reveals six homologs and one active gene copy. Plant Cell 10, 1055-1068. doi: 10.1105/tpc. 10.6.1055

Song, J., Bradeen, J. M., Naess, S. K., Raasch, J. A., Wielgus, S. M., Haberlach, G. T., et al. (2003). Gene RB cloned from Solanum bulbocastanum confers broad spectrum resistance to potato late blight. Proc. Natl. Acad. Sci. U.S.A. 100, 9128-9133. doi: 10.1073/pnas.1533501100

Tameling, W. I., and Baulcombe, D. C. (2007). Physical association of the NBLRR resistance protein $\mathrm{Rx}$ with a Ran GTPase-activating protein is required for extreme resistance to Potato virus X. Plant Cell 19, 1682-1694. doi: 10.1105/ tpc.107.050880

Tian, D., and Babadoost, M. (2004). Host range of Phytophthora capsici from pumpkin and pathogenicity of isolates. Plant Dis. 88, 485-489. doi: 10.1094/ PDIS.2004.88.5.485

Tomita, R., Sekine, K. T., Mizumoto, H., Sakamoto, M., Murai, J., Kiba, A., et al. (2011). Genetic basis for the hierarchical interaction between Tobamovirus spp. and L resistance gene alleles from different pepper species. Mol. Plant Microbe Interact. 24, 108-117. doi: 10.1094/MPMI-06-10-0127

Tor, M., Gordon, P., Cuzick, A., Eulgem, T., Sinapidou, E., Mert-Turk, F., et al. (2002). Arabidopsis SGT1b is required for defense signaling conferred by several downy mildew resistance genes. Plant Cell 14, 993-1003. doi: 10.1105/tpc. 001123

van der Vossen, E., Sikkema, A., Hekkert, B. L., Gros, J., Stevens, P., Muskens, M. et al. (2003). An ancient R gene from the wild potato species Solanum bulbocastanum confers broad-spectrum resistance to Phytophthora infestans in cultivated potato and tomato. Plant J. 36, 867-882. doi: 10.1046/j.1365-313X. 2003.01934.x

Vega-Arreguin, J. C., Jalloh, A., Bos, J. I., and Moffett, P. (2014). Recognition of an Avr3a homologue plays a major role in mediating nonhost resistance to Phytophthora capsici in Nicotiana species. Mol. Plant Microbe Interact. 27, 770-780. doi: 10.1094/MPMI-01-14-0014-R

Wesley, S. V., Helliwell, C. A., Smith, N. A., Wang, M. B., Rouse, D. T., Liu, Q., et al. (2001). Construct design for efficient, effective and high-throughput gene silencing in plants. Plant J. 27, 581-590. doi: 10.1046/j.1365-313X.2001.01105.x

Wiermer, M., Feys, B. J., and Parker, J. E. (2005). Plant immunity: the EDS1 regulatory node. Curr. Opin. Plant Biol. 8, 383-389. doi: 10.1016/j.pbi.2005. 05.010

Xiao, S., Calis, O., Patrick, E., Zhang, G., Charoenwattana, P., Muskett, P., et al. (2005). The atypical resistance gene, RPW8, recruits components of basal defence for powdery mildew resistance in Arabidopsis. Plant J. 42, 95-110. doi: 10.1111/j.1365-313X.2005.02356.X

Yang, S., Gu, T., Pan, C., Feng, Z., Ding, J., Hang, Y., et al. (2008). Genetic variation of NBS-LRR class resistance genes in rice lines. Theor. Appl. Genet. 116, 165-177. doi: 10.1007/s00122-007-0656-4

Zuluaga, A. P., Vega-Arreguin, J. C., Fei, Z., Ponnala, L., Lee, S. J., Matas, A. J., et al. (2016). Transcriptional dynamics of Phytophthora infestans during sequential stages of hemibiotrophic infection of tomato. Mol. Plant Pathol. 17, 29-41. doi: $10.1111 /$ mpp. 12263

Conflict of Interest Statement: The authors declare that the research was conducted in the absence of any commercial or financial relationships that could be construed as a potential conflict of interest.

Copyright (c) 2017 Vega-Arreguín, Shimada-Beltrán, Sevillano-Serrano and Moffett. This is an open-access article distributed under the terms of the Creative Commons Attribution License (CC BY). The use, distribution or reproduction in other forums is permitted, provided the original author(s) or licensor are credited and that the original publication in this journal is cited, in accordance with accepted academic practice. No use, distribution or reproduction is permitted which does not comply with these terms. 